\title{
Plant Regeneration and Agrobacterium-Mediated Transformation of Achyranthes bidentata using Cotton EREBP Gene
}

\author{
Hong Ying Duan*, Xiao Sheng Ding, Jian Ying Song, Yun Long He and Yan Qing Zhou \\ College of Life Sciences, Henan Normal University, Henan Xinxiang 453007, P. R. China
}

\begin{abstract}
The aim of this work was to study the plant regeneration and Agrobacterium-mediated transformation of Achyranthes bidentata using cotton EREBP gene. Results showed that the callus induction rate of stems from A. bidentata was the highest (100\%) and bud was in approximately $70 \%$ of calli from stems. However bud differentiation rate of the callus from leaves and petioles was very low. Compared with ceftriaxone, 200mg/L cefotaxime could completely control Agrobacterium tumefaciens and had relatively less toxic action on the stems of A. bidentata. In addition, the induction rate of callus resistant to hygromycin was the highest when infected for 3 min and co-cultivated for $3 \mathrm{~d}$. Six positive transgenic plants transformed with pCAMBIA1304-GhEREB2 expression vector were obtained and confirmed by PCR. The expression of target gene GhEREB2 was detected in five transgenic plants by RT-PCR. In brief, an efficient system of genetic transformation and plant regeneration was established for A. bidentata.
\end{abstract}

Key words: Achyranthes bidentata, EREBP gene, Agrobacterium tumefaciens, genetic transformation

\section{INTRODUCTION}

Achyranthes bidentata belongs to Amaranthaceae family and is a perennial herb that mainly has originated from Qinyang, Wuzhi of Henan province in China. A. bidentata has many medicinal effects and is frequently used in health cure (Han et al. 2005; Jin et al. 2007; Chen et al. 2009; Zhu et al. 2012). It is not resistant to low temperature and drought, and is inadvisably cultivated in saline-alkali soil and low-lying land. The yield of $A$. bidentata is seriously reduced because growth and development are affected by the disease and insect, but the improvement in yield and quality of $A$. bidentata is only limited to the control of field management at present.
Along with the rapid development and extensive application of molecular biology, genetic engineering is used to enhance the resistance of plant and has become an effective approach in plant breeding for stress tolerance (BhatnagarMathur et al. 2008; Cominelli and Tonelli 2010; Mallikarjuna et al. 2011; Li et al. 2012). Studies of transgenic medicinal plants are also remarkable and good achievements have been obtained in improving the resistance, adaptability and quality of medicinal plants (Zhou et al. 2009; Pandey et al. 2010). However, there is no report on the genetic transformation of $A$. bidentata. The resistance to disease and environment stress is usually controlled by many genes, while one transcription factor could regulate expression of many relevant genes. Therefore, compared with transferring

*Author for correspondence: dxdhy@126.com 
individual function gene to increase the resistance of plant, it is more efficient to modify or enhance the regulation of one transcription factor in molecular plant breeding.

EREBP transcription factor is only found in plant, closely related with response to drought, high salt, low temperature, disease and plant development. Simultaneously it participates in many signal pathways, such as growth and development, disease and environment stress (Riechmann and Meyerowitz 1998). GhEREB2 gene was isolated from cotton (Gossypium hirsutum L.) with yeast one-hybrid system by Duan et al (2006). Its encoding protein has a highly conserved AP2/EREBP domain and other typical features of EREBP transcription factors. The cDNA sequence of GhEREB2 has been submitted to GenBank databases with GenBank accession numbers AY962571. In addition, GhEREB2 was expressed in the roots, stems and leaves of cotton, and expression of GhEREB2 were induced by ethylene and jasmonic acid, also slightly induced by stress conditions, such as drought and cold treatment. Further studies indicated that GhEREB2 might be involved as the positive transcription factor in biotic and abiotic stresses signal transduction pathways (Duan et al. 2006). In this work, the cDNA sequence of GhEREB2 was transferred to A. bidentata by Agrobacterium tumefaciens in order to establish a set of low-cost, adaptable and efficient genetic transformation system for $A$. bidentata and obtain some transgenic plants to provide germplasm resource for new cultivar breeding of A. bidentata.

\section{MATERIALS AND METHODS}

\section{Strain and plant material}

Agrobacterium tumefaciens GV3101 carrying pCAMBIA1304-GhEREB2 expression vector was available in our laboratory. It contained CaMV35S promoter, GhEREB2, GFP, GUS and Hyg genes in pCAMBIA1304-GhEREB2 plasmid. The seeds of $A$. bidentata were kindly provided by Jiaozuo Academy of Agricultural Sciences, Henan, P.R.China.

\section{Culture of aseptic seedlings}

The seeds of $A$. bidentata were soaked at $24{ }^{\circ} \mathrm{C}$ for $12 \mathrm{~h}$, surface-sterilized for $30 \mathrm{~s}$ with $75 \%$ ethanol, subsequently deep sterilized for 8 min with $0.1 \%$ mercury dichloride, and then washed five times with sterile water. The aseptic seeds were cultured on MS medium at $26 \pm 1{ }^{\circ} \mathrm{C}$ under $16 \mathrm{~h}$ photoperiod and a 1000-1200 lux light intensity.

\section{Dedifferentiation and redifferentiation of explants}

After the culturing of A. bidentata seedlings for 7 $\mathrm{d}$ on MS medium, leaves, petioles and stems were used as explants, separately cut into $0.25 \mathrm{~cm}^{2}$ or $0.5-1.0 \mathrm{~cm}$ fragments, and cultured on MS medium supplemented with $2.26 \mu \mathrm{M}$ 2,4-D and $2.69 \mu \mathrm{M}$ NAA to carry out the induction and differentiation of callus and plant regeneration. In every experiment, 100 explants were cultured and the experiments were repeated three times. The rates of callus induction from explants were calculated after 20 days of culture, and the rates of bud differentiation from callus were calculated after 30 days.

\section{Preparation of Agrobacterium tumefaciens liquid culture}

Agrobacterium tumefaciens carrying the expression vector kept at $-70{ }^{\circ} \mathrm{C}$ were inoculated in YEB solid medium supplemented with 50 $\mu \mathrm{g} / \mathrm{ml} \mathrm{Kan}$ and $40 \mu \mathrm{g} / \mathrm{ml}$ Rif, and cultured at $28{ }^{\circ} \mathrm{C}$ for $2 \mathrm{~d}$ in the dark. Subsequently, single clone was inoculated in $5 \mathrm{ml}$ YEB liquid medium with 50 $\mu \mathrm{g} / \mathrm{ml} \mathrm{Kan}$ and $40 \mu \mathrm{g} / \mathrm{ml}$ Rif. After culturing under shaking conditions at $28{ }^{\circ} \mathrm{C}$ for $2 \mathrm{~d}$ in the dark, a little bacterium suspension was transferred to 50 $\mathrm{ml}$ YEB liquid medium with $50 \mu \mathrm{g} / \mathrm{ml}$ Kan and 40 $\mu \mathrm{g} / \mathrm{ml}$ Rif and cultured for $24 \mathrm{~h}$ at the above conditions. The bacterial biomass was seperated and then suspended in MS liquid medium to make $\mathrm{OD}_{600}$ be $0.6-0.8$.

\section{Bacteriostasis experiment}

After co-cultivation in the dark at $28{ }^{\circ} \mathrm{C}$ for $3 \mathrm{~d}$, stem segments of $A$. bidentata were respectively cultured on MS medium with 0 (the control group), 50, 100, 200, 300 and $400 \mathrm{mg} / \mathrm{L}$ ceftriaxone or cefotaxime at $25-28{ }^{\circ} \mathrm{C}$ under the light conditions indicated above. As culturing for 4 $\mathrm{d}$, the bacteriostasis effect of ceftriaxone and cefotaxime on Agrobacterium tumefaciens was calculated and analyzed according to the presence of Agrobacterium tumefaciens on the stems. Subsequently, the effects of ceftriaxone and cefotaxime on callus formation from stems were observed when cultured for $14 \mathrm{~d}$. There were 30 explants per treatment which was repeated three times, and the inhibitory rate of cephalosporins on 
Agrobacterium tumefaciens was calculated as follows: inhibitory rate $(\%)=(1-$ No. of stems with Agrobacterium / No. of experimental stems) $\times 100 \%$.

\section{Transformation and regeneration of plant}

The stems of $A$. bidentata were used as transformed explants, cut into $0.5-1.0 \mathrm{~cm}$ fragments and firstly cultured on MS medium for pre-culture for $2 \mathrm{~d}$. Then the stem segments were immerged into Agrobacterium tumefaciens and cultured under shaking in the dark at $28{ }^{\circ} \mathrm{C}$. Subsequently, infected stem segments were put on sterile filter paper to remove the excess of bacterium suspension and separately cultured on MS medium in the dark at $25-28{ }^{\circ} \mathrm{C}$, then were transferred onto MS medium supplemented with $200 \mathrm{mg} / \mathrm{L}$ cefotaxime and cultured at $25-28{ }^{\circ} \mathrm{C}$ for $5 \mathrm{~d}$ under the light conditions indicated above. After that, the stem segments were grown on MS medium supplemented with $2.26 \mu \mathrm{M}$ 2,4-D, 2.69 $\mu \mathrm{M}$ NAA, $200 \mathrm{mg} / \mathrm{L}$ cefotaxime and $15 \mu \mathrm{g} / \mathrm{mL}$ hyg in order to induce the calli and different organs. When seedlings had well-developed root system and were higher than $2 \mathrm{~cm}$, they were carefully transferred in flowerpot and kept in culture.

In addition, after immersing into Agrobacterium tumefaciens for 1, 2, 3, 4 and $5 \mathrm{~min}$, the explants were cultured on MS medium for $3 \mathrm{~d}$, and then cultured on MS medium with $200 \mathrm{mg} / \mathrm{L}$ cefotaxime and $15 \mu \mathrm{g} / \mathrm{mL}$ hyg for four weeks in order to explore the effects of infection time. The effects of co-cultivation time were explored as following: after infected for $3 \mathrm{~min}$, the explants were separately cultured on MS medium for 2, 3, 4 and $5 \mathrm{~d}$, then transferred onto MS medium supplemented with $200 \mathrm{mg} / \mathrm{L}$ cefotaxime and 15 $\mu \mathrm{g} / \mathrm{mL}$ hyg for four weeks. Thirty explants were used in each experiment which was repeated three times.

\section{PCR}

Total genomic DNA extraction from the leaves of transgenic and non-transgenic A. bidentata was performed by CTAB (cetyltrimethyl ammonium bromide) extraction procedure. Plasmid DNA of pCAMBIA1304-GhEREB2 in Agrobacterium tumefaciens strain GV3101 was extracted with plasmid isolation system (BioTechnologies Engineering Co. Ltd, HuaMei, P.R.China), and the purity and quality of DNA were respectively determined by the spectrophotometry and denaturing agarose gel electrophoresis. The positive and negative controls were respectively plasmid DNA of pCAMBIA1304-GhEREB2 and genomic DNA of non-transgenic A. bidentata. The PCR reaction volume was $25 \mu \mathrm{l}$, which consisted of $5 \mu \mathrm{l} 10 \times$ PCR buffer, $0.5 \mu \mathrm{l} 10 \mathrm{mmol}^{-\mathrm{L}^{-1}}$ dNTP, $0.5 \mu 150$ mmol. $\mathrm{L}^{-1} \mathrm{Mg}^{2+}, 0.2 \mu 110 \mu$ mol.L 1 forward primer, $0.2 \mu 1 \quad 10 \mu$ mol. $L^{-1}$ reverse primer, $1 \mu 1$ DNA template, $0.25 \mu 15 \mathrm{U}_{\mu} \mathrm{l}^{-1} \mathrm{Taq}$ DNA polymerase and $17.35 \mu \mathrm{l} \mathrm{ddH}_{2} \mathrm{O}$, in which PCR primers of target gene were the following: forward primer 5'AACAAGATCTTATGTGTGGAGGTGCAATT ATTTC-3', reverse primer 5' ACCAACTAGTCTGTTGTTGATGATGTGTCA C-3', and were compounded by Sangon BioTechnologies Co. Ltd (Shang hai, P.R.China). The PCR amplification was performed according to the following procedure: $5 \mathrm{~min}$ of predenaturation at $95{ }^{\circ} \mathrm{C}$, followed by 35 cycles including $30 \mathrm{~s}$ at $94{ }^{\circ} \mathrm{C}, 45 \mathrm{~s}$ at $55^{\circ} \mathrm{C}, 1.5 \mathrm{~min}$ at $72{ }^{\circ} \mathrm{C}$, and finally $10 \mathrm{~min}$ of elongation at $72{ }^{\circ} \mathrm{C}$. The PCR products were separated by $1.0 \%$ agarose gel electrophoresis.

\section{RT-PCR}

Total RNA from the leaves of transgenic and nontransgenic A. bidentata was extracted by the total RNA isolation system (Promega Corporation, America) according to instructions of products Z5110 and Z5651. The yield and purity of total RNA were determined by spectrophotometry, and integrity of total RNA was determined by denaturing agarose gel electrophoresis. To study expression of target gene, RT-PCR analysis was performed. One microgram of total RNA was analyzed with One Step RNA PCR Kit (AMV) (TaKaRa biotechnology Co., Ltd. Japan) according to manufacturer's instruction. RT-PCR primers were the same as for PCR. RT-PCR products were separated by $1.2 \%$ agarose gel electrophoresis.

\section{RESULTS}

Factors influencing genetic transformation Effects of explant type in plant regeneration

When the leaves, petioles and stems of $A$. bidentata seedlings were cultured for $5 \mathrm{~d}$ on MS medium supplemented with 2,4-D and NAA, the cut of stem segments displayed expansion. Subsequently flaxen callus were formed at the cut 
of stem segments, some appeared in site of exposure between the stem segments and medium. The formation of callus peaked when the stem segments were cultured for $7 \mathrm{~d}$. Compared with the stems, callus initiation from the leaves was relatively later. The edge of most leaves curled when cultured for $6 \mathrm{~d}$. After $8 \mathrm{~d}$, callus were formed in some leaves and distributed in leaf vein and leaf edge, and the formation of callus peaked at $11 \mathrm{~d}$. Callus initiation from the petioles was close to that of the leaves and callus existed mainly at the cut of petioles. However, the formation of callus peaked at approximately $9 \mathrm{~d}$. Along with increase of the culture time, callus gradually enlarged. The callus induction rates of explants were calculated after 20 days of culture.

As shown in Table 1, the potential to form the callus was different in the leaves, petioles and stems: callus induction rate of stems was $100 \%$, but that of the leaves and petioles was lower $(65$ and $52 \%$, respectively). The callus from different explants were cut into pieces and continued to be cultured for $30 \mathrm{~d}$. It was found that $71.5 \%$ of calli from stems could differentiate and form buds, while bud differentiation rate of leaves was merely $0.3 \%$, and callus from petioles could not differentiate (Table 1).

Table 1 - Callus initiation and differentiation rate in the explants of A. Bidentata.

\begin{tabular}{lcc}
\hline Explants & Callus induction rate $(\%)$ & Bud differentiation rate $(\%)$ \\
\hline Stem & $100 \pm 2.4$ & $71.5 \pm 1.6$ \\
Leaf & $65.8 \pm 1.3$ & $0.3 \pm 0.01$ \\
Petiole & $52.2 \pm 1.4$ & 0.0 \\
\hline
\end{tabular}

Note: the callus induction rate was calculated after 20 days of culture, the bud differentiation rate was calculated after callus had been cultured for $30 \mathrm{~d}$.

\section{Inhibitory action of cephalosporins on Agrobacterium tumefaciens}

Antibiotics are required to inhibit the growth of Agrobacterium tumefaciens after co-cultivation, but antibiotics also partly inhibit the growth and differentiation of plant tissues. Therefore, the type and concentration of antibiotic must be selected. As shown in Table 2, the inhibitory action of ceftriaxone and cefotaxime on Agrobacterium tumefaciens was better when their concentration increased, in which the inhibitory action of cefotaxime was the best and growth of Agrobacterium tumefaciens could be completely controlled by $200 \mathrm{mg} / \mathrm{L}$ cefotaxime; higher concentration of 300 and $400 \mathrm{mg} / \mathrm{L}$ ceftriaxone also fully suppressed the growth of Agrobacterium tumefaciens. In addition, the cut of stem browned and did not expand when cultured for $7 \mathrm{~d}$ on MS medium with $200 \mathrm{mg} / \mathrm{L}$ ceftriaxone, while the growth of stems was fine on MS medium with 200 $\mathrm{mg} / \mathrm{L}$ cefotaxime and there was callus formation in some stems (Fig. 1). Hence, the toxic action of cefotaxime on $A$. bidentata was relatively rather little.

\section{Effect of infection time}

As well-known, the infection time of explants by
Agrobacterium tumefaciens is the key for transformation. Short time of infection is not beneficial for the invasion of Agrobacterium tumefaciens, but too long time of infection might hurt the explant and even result in necrosis, thus the efficiency of T-DNA transfer into explant would be reduced. In this study, when the explants were infected for $3 \mathrm{~min}$, callus was initiated from approximately $78 \%$ explants, but the induction rate of the resistant callus was $27-45 \%$ when infected for 1-2 min; furthermore it was lower (23\% or so) when the explants were infected for 4-5 min. Therefore, the infection for $3 \mathrm{~min}$ by Agrobacterium tumefaciens was optimum for the transformation of $A$. bidentata.

\section{Effect of co-cultivation time}

Because co-cultivation could weaken the growth of explant and influence the transformation rate, suitable time of co-cultivation is necessary to one good system of genetic transformation. In this study, the induction rate of resistant callus first increased and then decreased with the increase of co-cultivation time. It was the highest (78.5\%) when co-cultivated for $3 \mathrm{~d}, 55 \%$ after $4 \mathrm{~d}$ of cocultivation and approximately $30 \%$ when cocultivated for $2 \mathrm{~d}$ or $5 \mathrm{~d}$. 
Table 2- Inhibitory action of cefotaxime and ceftriaxone on Agrobacterium tumefaciens.

\begin{tabular}{lcc}
\hline \multirow{2}{*}{ Concentration(mg/L) } & \multicolumn{2}{c}{ Inhibition rate $(\%)$} \\
\cline { 2 - 3 } & Cefotaxime & Ceftriaxone \\
\hline 0 & 0 & 0 \\
100 & 0 & 0 \\
200 & 63.33 & 0 \\
300 & 100 & 84 \\
400 & 100 & 100 \\
\end{tabular}
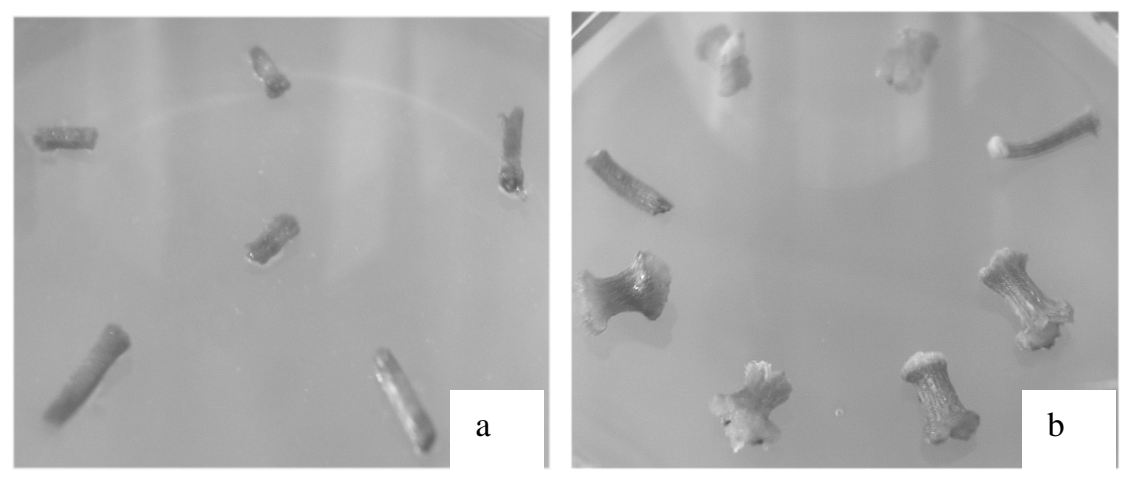

Figure 1 - Effects of cephalosporins on growth and callus induction of A. bidentata stems explants Stems cultured for $7 \mathrm{~d}$ on MS medium with $200 \mathrm{mg} / \mathrm{L}$ ceftriaxone (a) or cefotaxime (b).

\section{Regeneration of transgenic plants}

The induction and differentiation of the callus from the stem segments infected by Agrobacterium tumefaciens and cultured for $50 \mathrm{~d}$ on MS medium supplemented with plant growth regulators, $200 \mathrm{mg} / \mathrm{L}$ cefotaxime and $15 \mu \mathrm{g} / \mathrm{mL}$ hyg, are shown in Figure 2 ( $a$ and $b$ ). A total of fifteen resistant buds were obtained. When these buds were cultured for $12 \mathrm{~d}$ on MS medium
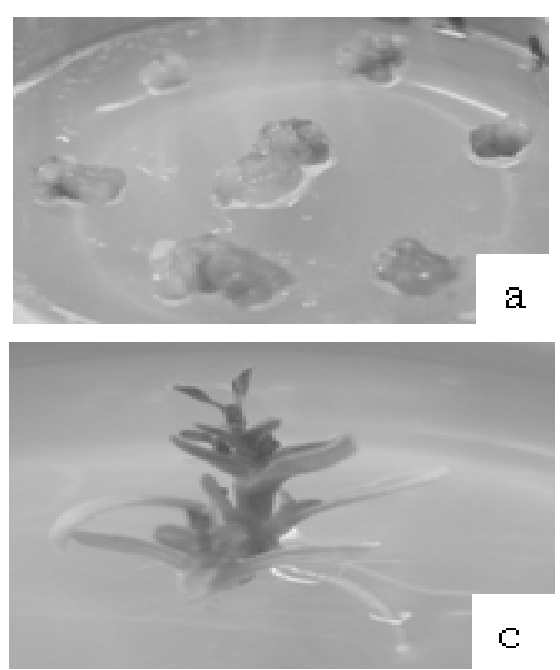

supplemented with cefotaxime and hygromycin, the adventitious roots were observed at the basal part of stems (Fig. 2, c and d), and all the shoots produced roots within 2-3 weeks of culture. Nine transgenic seedlings with developed root system were obtained after 4-5 weeks, which were carefully transferred into flowerpot when they were higher than $2 \mathrm{~cm}$ and kept in the glasshouse.
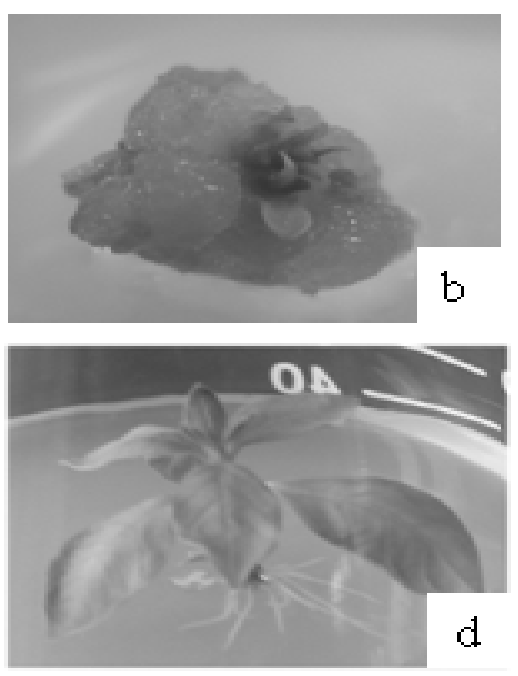

Figure 2 - Regeneration of of A. bidentata transgenic plants resistant to hygromycin Callus (a), bud (b), rooting of bu (c) and plant (d). 


\section{Molecular determination of transgenic plants PCR detection}

The genomic DNA of the transgenic and nontransgenic A. bidentata and plasmid DNA were respectively extracted by the CTAB method and plasmid isolation system. The purity and quality of DNA were high and banding pattern of DNA was intact. As shown in Figure 3, the 780bp target DNA fragment could be amplified from the pCAMBIA1304-GhEREB2 plasmid DNA and genomic DNA of six transgenic plants. Nevertheless the target DNA fragment was not amplified from the genomic DNA of nontransformed plants and other three putative transformed plants. Thus, it was preliminary proved that GhEREB2 gene was integrated into $A$. bidentata genome.

\section{RT-PCR analysis}

Total RNA from the leaves of transgenic and nontransgenic A. bidentata was extracted. The yield and purity of total RNA were high and the banding pattern of RNA was intact. Since Northern blot analysis produced only weak or undetectable signals (result not shown), the expression of target gene was analyzed by RT-PCR. As revealed in Figure 4, the 780bp target fragment was detected from the total RNA of five transgenic plants, but not found in one transgenic plant and nontransgenic plants. Accordingly, it indicated that GhEREB2 gene could be normally transcribed into RNA in five transgenic plants.

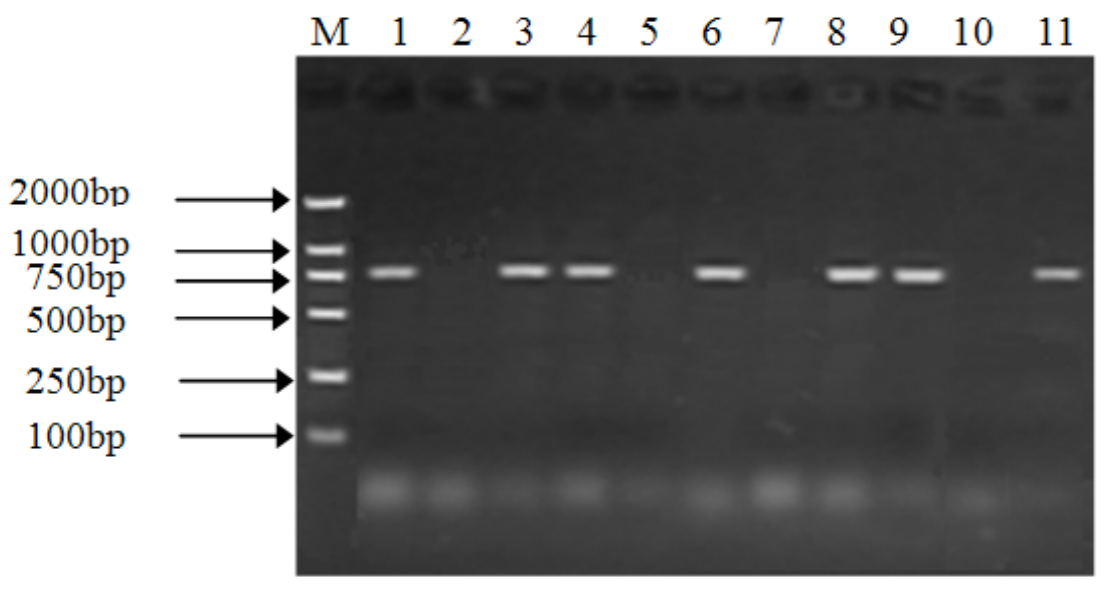

Figure 3 - PCR detection of $A$. bidentata transgenic plants

M. DNA marker, 1. positive control, 2. negative control and 3-11. putative transgenic plants.

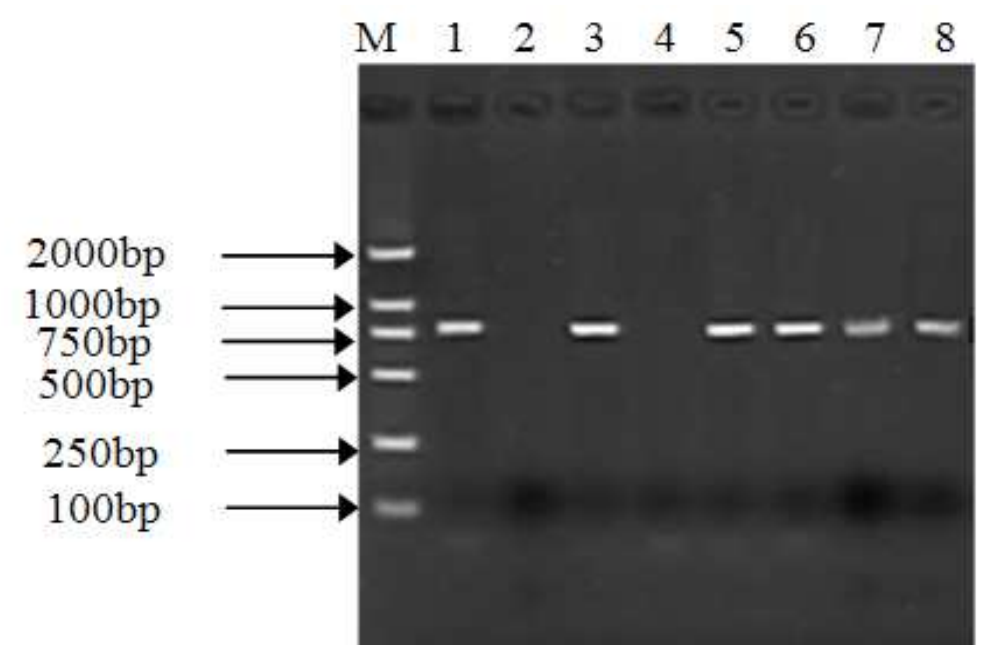

Figure 4 - RT-PCR analysis of target gene in A. bidentata transgenic plants

M. DNA Marker, 1. pCAMBIA1304-GhEREB2 plasmid DNA, 2. non-transgenic plant and 3-8. putative transgenic plants. 


\section{DISCUSSION}

In plant genetic transformation, the important premise is to establish the efficient and stable system of plant regeneration and regeneration rate of explant should be $80-90 \%$ in order to assure high transformation rate. In this study, the leaves, petioles and stems of $A$. bidentata could induce and form callus, but there was tremendous difference in initiation time and induction rate of callus. Compared with the leaves and petioles, the highest induction rate of callus was observed from the stems of A. bidentata. Li et al (2000) found that leaves, stems and bulbil of Dioscorea opposita formed callus, but the potential of different explants to form callus was distinct, indicating that the dedifferentiation potential of different parts in a plant was diverse. Furthermore, the redifferentiation of callus from different explants of $A$. bidentata displayed great diversity, callus from stems could easily differentiate bud, but the differentiation of callus from leaves was relatively low, especially the callus from petioles failed to divide into bud. However, the petiole of Populus sp had strong differentiation potential (Jafari et al. 1995). On the basis of totipotency theory, the explants from different parts of plant can differentiate and develop, however the differentiation potential appears to be variable because cell structures, physiological status and levels of endogenous hormones in all kinds of organs also are various (Chovelon et al. 2011). Therefore, the explants should be selected to establish the system of plant regeneration and genetic transformation.

As it is well known, genetic transformation mediated by Agrobacterium tumefaciens is a complex process and transformation efficiency is affected by many factors, in which the type and concentration of antibiotics is very important to enhance the frequency of genetic transformation. Cephalosporins have been generally used in genetic transformation. Yang et al (2011) found $200 \mathrm{mg} / \mathrm{L}$ cefotaxime as optimal to suppress Agrobacterium tumefaciens. In the present work $200 \mathrm{mg} / \mathrm{L}$ cefotaxime also could completely control Agrobacterium tumefaciens and had relatively less hurt on $A$. bidentata. In addition, the suitable time of infection and co-cultivation are also important to establish a good system of genetic transformation (Karmarkar 2001), but optimal time of infection depends on the explant type because their sensitivity to Agrobacterium tumefaciens varies. In this study, the induction rate of resistant callus was the highest when the explants were infected by Agrobacterium tumefaciens for $3 \mathrm{~min}$, but was lower when infected for 1-2 min or 4-5 min, indicating the poor effectiveness of infection when the explants were infected for short time by Agrobacterium tumefaciens, while longer time of infection would hurt explants and also reduce the induction rate of resistant callus. Besides, the induction rate of resistant callus was the highest when co-cultivated for $3 \mathrm{~d}$, which also demonstrated that cocultivation time influenced the transformation rate and the co-cultivation of $3 \mathrm{~d}$ was suitable.

In this study, six transgenic A. bidentata plants were obtained, while the expression of target gene was only detected in five transgenic plants by RTPCR. The abnormal expression of target gene in the transgenic plant could be due to many factors (Capecchi 1989; Prins and Goldach 1996; Stav et al. 2010; Zhang and Rossi 2011). Several mechanisms have been reported leading to gene silencing, such as position-dependent gene silencing (PDGS), transcriptional gene silencing (TGS) and post-transcriptional gene silencing (PTGS) (Ratcliff et al. 1998; Scherer and Rossi 2003). In this study, the target gene was not normally expressed in one transgenic plant, inferring that the gene silencing might have happened, but the mechanism of this gene silencing still needs to be further analyzed and discussed.

In summary, an efficient system of genetic transformation and plant regeneration in $A$. bidentata was established and some transgenic $A$. bidentata plants were obtained, which could provide feasible theoretical basis for genetic transformation and new germplasm breeding of $A$. bidentata.

\section{ACKNOWLEDGMENTS}

This work was kindly supported by the Science Fund from Henan province (2008A180015), (2008B180007) and (12A180015) and grant of young teachers in Henan province institution of higher learning (2009GG J S2045), in P. R. China. 


\section{REFERENCES}

Bhatnagar-Mathur P, Vadez V, Sharma KK. Transgenic approaches for abiotic stress tolerance in plants: retrospect and prospects. Plant Cell Rep. 2008; 27(3): 411-424.

Capecchi MR. Altering the genome by homologous recombination. Science, 1989; 244: 1288-1292.

Chen Q, Liu Z, He JH. Achyranthes bidentata polysaccharide enhances immune response in weaned piglets. Immunopharmacol Immunotoxicol. 2009; 31(2): 253-260.

Chovelon V, Restier V, Giovinazzo N, Dogimont C, Aarrouf J. Histological study of organogenesis in Cucumis melo L. after genetic transformation: why is it difficult to obtain transgenic plants? Plant Cell Rep. 2011; 30(11): 2001-2011.

Cominelli E, Tonelli C. Transgenic crops coping with water scarcity. N Biotechnol. 2010; 27(5): 473-477.

Duan HY, Li FG, Wu XD, Ma DM, Wang M, Hou YX. Cloning and characterization of two EREBP transcription factors from Cotton (Gossypium hirsutum L.). Biochemistry (Mosc). 2006; 71(3): 285293.

Han SB, Lee CW, Yoon YD, Lee JH, Kang JS, Lee $\mathrm{KH}$, et al. Prevention of arthritic inflammation using an oriental herbal combination BDX-1 isolated from Achyranthes bidentat and Atractylodes japonic. Arch Pharm Res. 2005; 28(8): 902-908.

Jafari MA, Kiss J, Gergácz J, Heszky LE. High efficiency callus induction and plant regeneration in petiole culture of four poplar genotypes. Acta Biol Hung. 1995; 46(1): 51-59.

Jin LQ, Zheng ZJ, Peng Y, Li WX, Chen XM, Lu JX. Opposite effects on tumor growth depending on dose of Achyranthes bidentat polysaccharides in C57BL/6 mice. Int Immunopharmacol. 2007; 7(5): 568-577.

Karmarkar SH. Genetic transformation and hairy root induction in Holostemma ada-kodien K. Schum-a vulnerable medicinal plant. Indian J Exp Biol. 2001; 39(12): 1263-1267.

Li M, Li Y, Li H, Wu G. Improvement of paper mulberry tolerance to abiotic stresses by ectopic expression of tall fescue FaDREB1. Tree Physiol. 2012; 32(1): 104-113.

Li MJ, Xue JP, Chen MX, Guo MY, Qiu WH, Zhang JB. The influence of different factors on callus induction of Dioscorea opposita. Guihaia. 2000; 20(2): 156-160.

Mallikarjuna G, Mallikarjuna K, Reddy MK, Kaul T. Expression of OsDREB2A transcription factor confers enhanced dehydration and salt stress tolerance in rice (Oryza sativa L.). Biotechnol Lett. 2011; 33(8): 1689-1697.
Pandey V, Misra P, Chaturvedi P, Mishra MK, Trivedi PK, Tuli R. Agrobacterium tumefaciens-mediated transformation of Withania somnifera (L.) Dunal: an important medicinal plant. Plant Cell Rep. 2010; 29(2): 133-141.

Prins M, Goldach R. RNA-mediated virus resistance in transgenic plants. Arch Virol. 1996; 141(12): 22592276.

Ratcliff FG, MacFarlane SA, Baulcombe DC. Gene silencing without DNA: RNA-mediated crossprotection between virus. Plant Cell. 1999; 11(7): 1207-1216.

Riechmann JL, Meyerowitz EM. The AP2/EREBP family of plant transcription factors. Biol Chem. 1998; 379(6): 633-646.

Scherer LJ, Rossi JJ. Approaches for the sequencespecific knockdown of mRNA. Nat Biotechnol. 2003; 21: 1457-1465.

Stav R, Hendelman A, Buxdorf K, Arazi T. Transgenic expression of tomato bushy stunt virus silencing suppressor P19 via the $\mathrm{pOp} / \mathrm{LhG} 4$ transactivation system induces viral-like symptoms in tomato. Virus Genes. 2010; 40(1): 119-129.

Yang J, Bi HP, Fan WJ, Zhang M, Wang HX, Zhang P. Efficient embryogenic suspension culturing and rapid transformation of a range of elite genotypes of sweet potato (Ipomoea batatas [L.] Lam.). Plant Sci. 2011; 181(6): 701-711.

Zhang X, Rossi JJ. Phylogenetic comparison of small RNA-triggered transcriptional gene silencing. $\mathrm{J}$ Biol Chem. 2011; 286(34): 29443-29448.

Zhou YQ, Duan HY, Zhou CE, Li JJ, Gu FP, Wang F, et al. Hairy root induction and plant regeneration of Rehmannia glutinosa Libosch. f. hueichingensis Hsiao via Agrobacterium rhizogenes-mediated transformation. Russ J Plant Physiol. 2009; 56(2): 224-231.

Zhu X, Pan Y, Zheng L, Cui L, Cao Y. Polysaccharides from the Chinese medicinal herb Achyranthes bidentata enhance anti-malarial immunity during Plasmodium yoelii 17XL infection in mice. Malar $J$. 2012; 11: 49. 